\title{
Sauber und sicher - Optimierte Oberflächen für den ÖPNV
}

\author{
Die Anforderungen für Lacke im Innenraum von Bus und Bahn sind seit jeher hoch. In Zeiten der \\ Corona-Pandemie müssen die Oberflächen zudem den neuen hygienischen Standards gerecht \\ werden und sollen dabei nicht nur verstärkten Reinigungszyklen standhalten, sondern auch selbst \\ aktiv dazu beitragen, die Verbreitung von Mikroorganismen zu verringern.
}

Millionen von Fahrgästen nutzen täglich die öffentlichen Transportmittel weltweit. Haltegriffe, Armstützen, Rückenlehnen und viele weitere High Touch Areas werden dabei Millionen Mal berührt. Um die Fahrgäste zu schützen und ihnen wieder ein sicheres und möglichst unbeschwertes Fahrerlebnis zu ermöglichen, sind neben den Betreibern auch die Hersteller gefragt: Neue Hygienekonzepte und Technologien sollen Reisen zukünftig noch sauberer und sicherer machen und das Vertrauen der

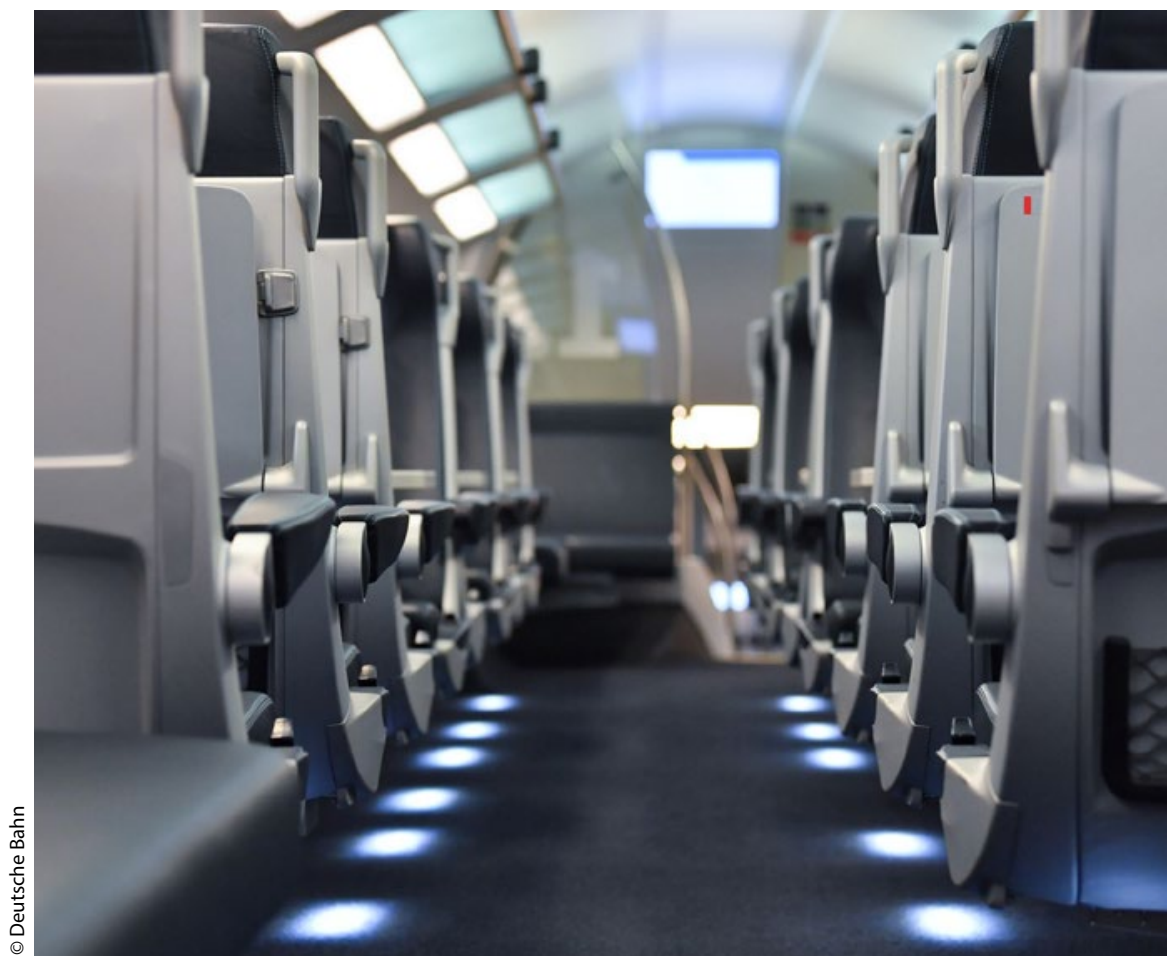

Haltegriffe, Armstützen und Rückenlehnen werden in öffentlichen Transportmitteln Millionen Mal berührt und stellen damit hohe Anforderungen an die Sauberkeit.
Fahrgäste in den Personennahverkehr wieder stärken.

Mankiewicz bietet antimikrobielle Lacksysteme für den Fahrgastinnenraum an, die einen aktiven Beitrag zur Optimierung von schlüssigen Hygienekonzepten leisten. Die 2K-Lacke der Lackreihe „Alexit Saniseries“ enthalten antimikrobielle Wirkstoffe, die das Wachstum von Mikroorganismen auf der beschichteten Oberfläche hemmen.

Die Wirksamkeit des Lacksystems ist nach dem international anerkannten JIS Z 2801 Standard geprüft. Hierbei wurde die Klassifizierung „Log-Stufe 4“ erreicht, was einer Bakterienreduktion von 99,99\% entspricht. Die Abtötung von Bakterien leistet einen wertvollen Beitrag zur Hygiene in Zeiten von COVID-19: Es hat sich gezeigt, dass die Wirkung bestimmter Viren steigt, wenn der Gesundheitszustand von Personen bereits schlecht ist oder wenn eine bakterielle Infektion vorliegt. Durch die Verringerung der Bakterienlast wird daher indirekt auch der Schutz vor Viruserkrankungen verbessert.

\section{Verlängerte Reinigungs- und Desinfektionszyklen}

Mit der antimikrobiellen Wirkung unterstützt das Lacksystem die Betreiber auch bei der Reinigung: Da Mikroorganismen sich deutlich langsamer vermehren, können die Reinigungs- und Desinfektionszyklen 
Um eine große Farb- und Effektvielfalt zu bieten, besteht das Lacksystem aus einem

Deck- und einem Klarlack - so können nahezu alle gängigen Farb- und Designeffekte auch mit antimikrobieller Wirkung umgesetzt werden.

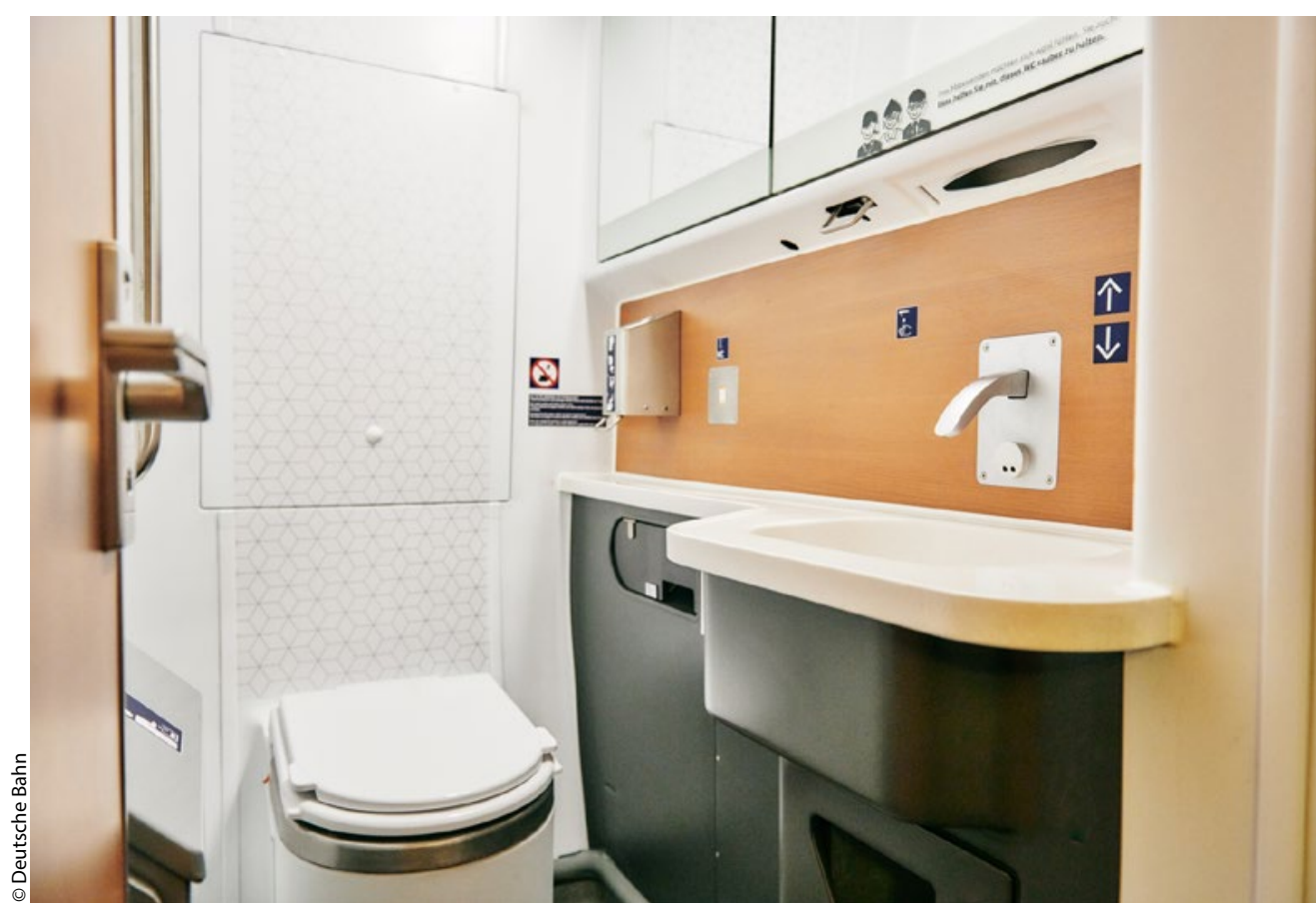

\section{Weitere Einsatzmöglichkeiten} Insbesondere in schwer $\mathrm{zu}$ säubernden Bereichen ist dies eine wertvolle Unterstützung. Schlecht zugängliche Ecken und Vertiefungen, die mit Desinfektionsmitteln nur eingeschränkt benetzt werden, erfahren durch die Lackierung trotzdem langfristigen Schutz. Tests mit ähnlichen Technologien von Mankiewicz beweisen auch nach 12 Jahren noch eine sehr hohe Wirksamkeit.

Um den Anwendern bei der Gestaltung von High Touch Areas weiterhin eine große Farb- und Effektvielfalt zu bieten, besteht das System aus einem Deck- und einem Klarlack - so können nahezu alle gängigen Farb- und Designeffekte auch mit antimikrobieller Wirkung umgesetzt werden. Das System ist außerdem in einer lösemittelbasierten und einer wasserbasierten Variante erhältlich und lässt sich somit bequem an die Lackier- und Umweltanforderungen der Hersteller anpassen.
Neben dem Einsatz in Bereichen mit viel Fahrgastkontakt macht das System auch noch an anderen Stellen Sinn, beispielsweise in Lüftungskanälen oder Klimaanlagen. Diese Bereiche können teilweise überhaupt nicht gereinigt werden und bieten deshalb einen Nährboden für Bakterien. Dadurch entstehen nicht nur unangenehme Gerüche, die dort befindlichen Mikroorganismen werden durch die Klimaanlage auch im gesamten Innenraum verteilt. Mit einer Beschichtung mit antimikrobiell wirkenden Inhaltsstoffen wird das Bakterienwachstum effektiv bekämpft, was die Klimaanlagen deutlich hygienischer macht.

Neben der Bahnbranche bildet auch die Medizintechnik einen vielversprechenden Einsatzort für Lacksysteme mit antimikrobiell wirksamen Inhaltsstoffen, denn Hygiene und Gesundheit spielen dort na- turgemäß eine besonders große Rolle. Außerdem könnten die Lacke in der Lebensmittelbranche Anwendung finden oder bei Haushaltsgeräten, in der System-Gastronomie, bei Geldautomaten und anderen stark frequentierten Bereichen des öffentlichen Lebens eingesetzt werden - der Vielfalt der Einsatzmöglichkeiten sind keine Grenzen gesetzt. //

\section{Kontakt}

Mankiewicz Gebr. \& Co.

Hamburg

rail@mankiewicz.com

www.mankiewicz.com

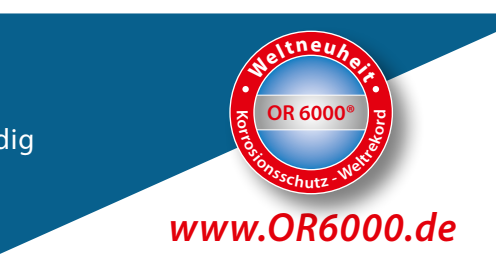

